\title{
Evaluation of the effect of $10 \%$ lidocaine spray on reducing the pain of intrauterine device insertion: A randomised controlled trial
}

\author{
M Hajiesmaello, ${ }^{1}$ MS (Midwifery); E Mohammadi, ${ }^{2} \mathrm{MD}$ (Obstetrics); H Farrokh-Eslamlou, ${ }^{3} \mathrm{MD}, \mathrm{PhD}$ \\ ${ }^{1}$ Department of Midwifery, Faculty of Nursing and Midwifery, Islamic Azad University, Urmia, Iran \\ ${ }^{2}$ Diabetes Research Centre, Health Research Institute, Ahvaz Jundishapur University of Medical Sciences, Ahvaz, Iran \\ ${ }^{3}$ Reproductive Health Research Center, Urmia University of Medical Sciences, Urmia, Iran
}

Corresponding author: $M$ Hajiesmaello (mhajiesmaello@yahoo.com)

\begin{abstract}
Background. The intrauterine device (IUD) is among the most efficient contraceptive methods. However, IUD insertion is accompanied by pain and discomfort.

Objectives. To evaluate the analgesic effects of $10 \%$ lidocaine spray in reducing pain during IUD insertion.

Method. In a randomised clinical trial, 80 volunteers attending two clinics for IUD insertion were selected for study, and randomly allocated to two groups. The intervention group received four puffs of $10 \%$ lidocaine spray on their cervix prior to IUD insertion. The routine procedure (without an analgesic) was followed in the control group. The intensity of perceived pain in both groups was measured using a visual analogue scale from 0 to 10 .

Results. The two groups had significant differences in pain intensity at all stages of the procedure $(p<0.001)$. The most painful stage of the procedure was tenaculum placement (mean (standard deviation) pain intensity 2.2 (1.34) in the intervention group; 4.25 (1.92) in the control group).

Conclusion. Based on our findings, 10\% lidocaine spray can be applied as a non-invasive, inexpensive, easy-to-use and accessible method to decrease IUD insertion pain.

S Afr J Obs Gynae 2019;25(1):25-29. DOI:10.7196/SAJOG.2019.v25i1.1383
\end{abstract}

Long-acting reversible contraception methods, such as intrauterine devices (IUDs), implants and injectable contraceptives, are associated with high effectiveness in preventing unintended pregnancies, and thereby their significant health and economic consequences. $^{[1]}$ International reports have demonstrated that IUDs are the second-most common contraceptive method worldwide, used by $14 \%$ of women of reproductive age who are married or in a union; the highest prevalences of IUD use in this demographic have been reported in China (over 40\%), South Korea and Uzbekistan. ${ }^{[2]}$ IUD prevalence in Iran was calculated as $8.1 \%$ in $2012 .{ }^{[2]}$ IUD insertion is generally associated with fear, which may decrease its acceptability, and anticipated pain or discomfort at the time of insertion could be a major barrier to the acceptance of IUDs among both clients and healthcare providers. ${ }^{[3,4]}$ Nulliparity, non-lactation and time since the last pregnancy have all been suggested as factors that increase the probability of pain and discomfort during the IUD insertion procedure. ${ }^{[5-7]}$

Previous studies have shown conflicting results regarding the effects of several pain-relief methods in IUD insertion. ${ }^{[8]}$ Randomised controlled trials have shown neither ibuprofen ${ }^{[9]}$ nor naproxen $^{[10,11]}$ to have beneficial effects on IUD insertion pain. Similarly, different doses of sublingual and vaginal misoprostol (also a nonsteroidal anti-inflammatory drug (NSAID) pain-relief methods) failed to reduce pain during IUD insertion. ${ }^{[6,12]}$

Widespread use of lidocaine gel has been reported from countries such as the $U K,{ }^{[13]}$ where different forms of intracervical lidocaine have been applied to attempt to decrease IUD insertion pain. However, studies on the effect of lidocaine gel have had conflicting results. In one of these, the effect of intracervical application of $2 \%$ lignocaine (lidocaine gel) on pain and discomfort perception on IUD insertion was studied, and showed some promising results. ${ }^{[14]}$ Two other randomised controlled trials, however, concluded that intracervical lidocaine gel did not decrease IUD insertion pain. ${ }^{[15,16]}$ Using a $1 \%$ lidocaine solution for paracervical anaesthesia similarly had no effect on pain. ${ }^{[17]}$

Because of its rapid absorption and distribution over a wide area, it may be expected that lidocaine in spray form would result in better anaesthesia. A study by Aksoy et al ${ }^{[18]}$ in Turkey reported a significant decrease in IUD insertion pain when using lidocaine $10 \%$ spray. We therefore hypothesised that $10 \%$ lidocaine spray (the only available concentration) would reduce IUD insertion pain in our study sample. The present study was performed to assess the analgesic impact of cervical lidocaine spray on IUD insertion pain.

\section{Methods}

The present randomised controlled trial used a parallel design on women who were referred for IUD insertion to the Reproductive Health Research Center in Urmia, Iran. Study participants were selected between March 2013 and September 2014. According to a sample size calculation, based on a $10 \%$ dropout rate, with a onesided $\alpha$ of $1 \%$, and $80 \%$ power, a total of 40 women in each trial group was needed. Parous women between 18 and 45 years of age were recruited from two selected urban public health facilities. The ethics committee of Urmia Islamic Azad University of Medical Sciences approved the study protocol (ref. no P/05124284/92). The women were firstly counselled for a broad spectrum of reproductive 
health services, including family planning. Those willing to have an IUD (copperbearing Tcu380A) inserted, and gave informed consent, participated in the trial. The exclusion criteria were women with a history of allergic reaction to lidocaine, those who were pregnant or had had a pregnancy within the last 6 weeks, women with a history of pelvic inflammatory disease, abnormal uterine anatomy, uterine size $<6 \mathrm{~cm}$ or $>9 \mathrm{~cm}$, Wilson's disease, unexplained vaginal bleeding or cervical malignancy, and those who had received analgesics or narcotic agents during the past 24 hours. Before insertion of the IUD, baseline sociodemographic characteristics and reproductive and obstetric and gynaecological histories of the women were recorded on a data sheet.

A researcher who was not involved in the trial protocol prepared envelopes containing either A (intervention group) or B (control group) markers using a block randomisation scheme in a $1: 1$ ratio, in blocks of four. The participants were then randomly allocated to the intervention group (in which $10 \%$ lidocaine spray was applied to the ectocervix) or the control group (routine IUD insertion with no analgesia). Based on previous studies, the two most probable confounders for IUD insertion pain are parity and previous obstetric experience (women who have only had caesarean sections may be viewed as nulliparous with respect to IUD placement). ${ }^{[19]}$ In order to control these confounders, subjects were stratified by type of delivery (normal vaginal delivery or caesarean only) in random blocks.

The IUDs were inserted by four midwives with advanced training in family planning and $>5$ years' experience in IUD insertion, according to a standard IUD insertion protocol. The intervention group received local anaesthetic using four puffs of $10 \%$ lidocaine (each puff containing $10 \mathrm{mg}$ lidocaine) at the cervical area. There was a standardised 3-minute waiting period between the administration of the lidocaine spray and IUD insertion. Fig. 1 shows the flow of participants through the trial.

In the control group, the IUD was inserted without local anaesthetic (the routine IUD insertion procedure at the health centre). While the midwives were not blinded, data on pain severity were collected by a research assistant not involved in the procedure. A visual analogue scale (VAS) was used to record

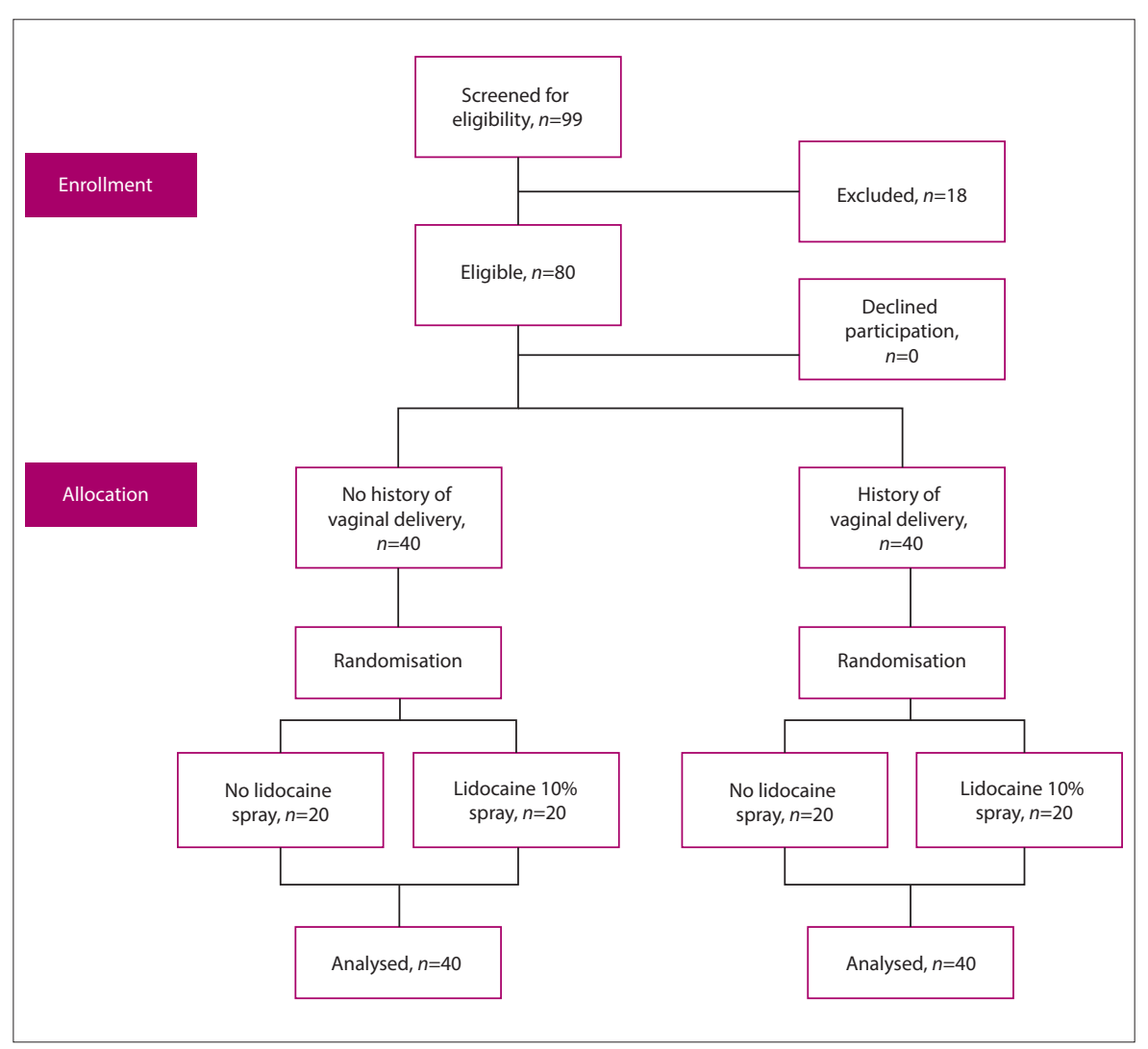

Fig.1. Consort diagram of flow of participants through the trial.

women's perceived pain at various points before, during and after the IUD insertion process. The scale was graded from 0 (no pain) to 10 (worst pain possible). Subjects rated their level of pain by making a mark on the VAS. Pain measurement was performed at baseline (when the woman's legs were positioned in stirrups), at three points during the procedure (tenaculum placement, uterine sounding and IUD insertion) and 15 minutes after the procedure.

Data were analysed using Statistical Package for Social Sciences 20.0 (SPSS Inc., USA). To evaluate differences between the two groups, Fisher's exact test and the independent sample $t$-test were used for categorical and normally distributed continuous variables, respectively. Statistical significance was set at $p \leq 0.05$. Repeated analysis of variance (ANOVA) measures were performed to identify intergroup differences in pain at baseline and during and after IUD insertion.

\section{Results}

We randomised a total sample of 80 women to receive Tcu380A IUDs either with or without $10 \%$ lidocaine spray as an intervention, at the Reproductive Health
Research Center of Urmia University of Medical Sciences in Urmia, Iran. None of the approached women declined participation and all had successful IUD insertions. The mean ages of the participants in the control and intervention group were not statistically different [30.6 (6.8) v. 28.8 (7.5); $p=0.26]$. The majority $(91.3 \%)$ of the participants did not have a high school diploma, and most were unemployed (95\%). The mean (SD) body mass indexes (BMIs) of women in the control and intervention groups were very similar (27.3 (3.3) v. 27.0 (4.2); $p=0.68$ ). Randomisation was successful for most variables including age, education, BMI, occupation, gravidity, number of children living and abortion history. The two groups were also similar with regard to current breastfeeding status, history of previous IUD use and history of cryosurgery on the cervix (Table 1).

The IUD was inserted during the menstruation period in most cases, except for four women from the intervention group and six from the control group, owing to breastfeeding amenorrhoea. The most painful phase of IUD insertion in both groups was tenaculum placement. The mean (SD; range) score of pain at this stage was $4.25(1.92 ; 1-8)$ in the control 
RESEARCH

Table 1. Distribution of study participants by selected demographic and reproductive characteristics $(N=80)$

\begin{tabular}{|c|c|c|c|}
\hline \multirow[b]{2}{*}{ Variable } & \multicolumn{2}{|c|}{ Group } & \multirow[b]{2}{*}{$p$-value } \\
\hline & Control $(n=40)$ & Intervention $(n=40)$ & \\
\hline Age (years), mean (SD) & $30.6(6.8)$ & $28.8(7.5)$ & 0.26 \\
\hline \multicolumn{4}{|l|}{ Education level, $n$ (\%) } \\
\hline$<12$ grades completed & $25(62.5)$ & $24(60.0)$ & \multirow[t]{3}{*}{$0.88^{*}$} \\
\hline 12 grades completed & $11(27.5)$ & $13(32.5)$ & \\
\hline University & $4(10.0)$ & $3(7.5)$ & \\
\hline \multicolumn{4}{|l|}{ Occupation, $n$ (\%) } \\
\hline Housewife & $38(95.0)$ & $38(95.0)$ & \multirow[t]{2}{*}{$1.0^{*}$} \\
\hline Employed & $2(5.0)$ & $2(5.0)$ & \\
\hline BMI, mean (SD) & $27.3(3.3)$ & $27.0(4.2)$ & 0.68 \\
\hline \multicolumn{4}{|l|}{ Number of previous abortions, $n$ (\%) } \\
\hline 0 & $31(77.5)$ & $28(70.0)$ & \multirow[t]{3}{*}{$0.64^{*}$} \\
\hline 1 & $7(17.5)$ & $8(30.0)$ & \\
\hline 2 & $2(5.0)$ & $4(10.0)$ & \\
\hline \multicolumn{4}{|l|}{ Parity } \\
\hline 1 & $12(30.0)$ & $12(30.0)$ & \multirow[t]{3}{*}{0.86} \\
\hline 2 & $23(57.5)$ & $21(52.5)$ & \\
\hline 3 & $5(12.5)$ & $7(17.5)$ & \\
\hline \multicolumn{4}{|l|}{ Number of previous deliveries, $n$ (\%) } \\
\hline \multicolumn{4}{|l|}{ NVD, with or without CS } \\
\hline 0 & $20(50.0)$ & $20(50.0)$ & \multirow[t]{4}{*}{$0.92^{*}$} \\
\hline 1 & $6(15.0)$ & $8(20.0)$ & \\
\hline 2 & $11(27.5)$ & $9(22.5)$ & \\
\hline 3 & $3(7.5)$ & $3(7.5)$ & \\
\hline \multicolumn{4}{|l|}{ Only CS } \\
\hline 0 & $18(45.0)$ & $16(40.0)$ & \multirow[t]{4}{*}{$0.73^{*}$} \\
\hline 1 & $9(22.5)$ & $11(27.5)$ & \\
\hline 2 & $12(30.0)$ & $10(25.0)$ & \\
\hline 3 & $1(2.5)$ & $3(7.5)$ & \\
\hline \multicolumn{4}{|l|}{ Number of living children, $n$ (\%) } \\
\hline 1 & $11(27.5)$ & $13(32.5)$ & 0.87 \\
\hline 2 & $23(57.5)$ & $21(52.5)$ & \\
\hline 3 & $6(15)$ & $6(15.0)$ & \\
\hline Months since last pregnancy, mean (SD) & $53.55(41.22)$ & $47.06(37.32)$ & 0.89 \\
\hline Months since last pregnancy, $n(\%)$ & & & \\
\hline 2 & $7(17.5)$ & $7(17.5)$ & $096^{*}$ \\
\hline $3-6$ & $7(17.5)$ & $6(15.0)$ & \\
\hline $7-12$ & $9(22.5)$ & $8(20.0)$ & \\
\hline $13-24$ & $3(7.5)$ & $5(12.5)$ & \\
\hline$>25$ & $14(35)$ & $14(35)$ & \\
\hline Currently lactating & & & \\
\hline No & $16(40)$ & $15(37.5)$ & $0.81^{\star}$ \\
\hline Yes & $24(60)$ & $25(62.5)$ & \\
\hline History of previous IUD use & & & \\
\hline No & $21(52.5)$ & $24(60)$ & $0.49^{*}$ \\
\hline Yes & $19(47.5)$ & $16(40)$ & \\
\hline The last method of contraception & & & \\
\hline Oral contraceptives & $15(37.5)$ & $11(27.5)$ & $0.50^{\star}$ \\
\hline Intrauterine device & $4(10)$ & $7(17.5)$ & \\
\hline Natural & $11(27.5)$ & $7(17.5)$ & \\
\hline Injection & $1(2.5)$ & $2(5)$ & \\
\hline Condom & $5(12.5)$ & $10(25.0)$ & \\
\hline Pregnancy & $4(10)$ & $3(7.5)$ & \\
\hline Current menses, days & & & \\
\hline No menstrual bleeding & $6(15)$ & $4(10)$ & $0.62^{*}$ \\
\hline 1 & $3(7.5)$ & $1(2.5)$ & \\
\hline 2 & $14(32)$ & $12(30)$ & \\
\hline 3 & $12(30)$ & $18(45)$ & \\
\hline$>4$ & $5(12.5)$ & $5(12.5)$ & \\
\hline
\end{tabular}


group and $2.20(1.96 ; 0-6)$ in the intervention group. During the hysterometer insertion, the mean (SD; range) scores for pain in the control and intervention groups were 3.45 (1.95; 0 - 8) and 1.92 (1.40; 0 - 5), respectively. During the IUD insertion, the mean (SD; range) pain score was $2.45(2.10 ; 0-8)$ in the control and $1.30(1.5 ; 0-5)$ in the intervention group $(p<0.001)$.

There was also a significant difference in pain score at 15 minutes after IUD insertion between the control and intervention groups (1.70 (1.75) v. 0.87 (1.04); $p=0.03)$. The intervention and control groups had no significant differences in background pain score (dysmenorrhoea) at IUD insertion time $(0.55$ (0.98) v. 0.47 (0.98); $p=0.38$ ) or anticipated pain before IUD insertion (5.02 (1.82) v. 4.35 (1.91); $p=0.6$ ).

The results of repeated measures of ANOVA suggested a significant difference in total scores of IUD insertion pain between the two groups $(p<0.001 ; \mathrm{F}=26.94)$. Systemic adverse effects of $10 \%$ lidocaine spray are very rare and are seen only at high doses $(>20$ puffs). As four puffs were used in the present study, no systemic adverse effects were observed (Table 2).

\section{Discussion}

The findings of the present study demonstrated that pain was felt in different phases of IUD insertion, including tenaculum placement and measurement of the uterine cavity with a hysterometer. Although pain during IUD insertion cannot be easily evaluated, as it is a subjective sensation and a combination of sensational, affective and cognitive elements, several studies on IUD insertion have reported pain of various levels, and noted that pain tolerance is also dependant on women's cultural background. ${ }^{[3,4]}$

The results showed tenaculum placement to be the most painful phase. While similar findings were reported by Seamark, ${ }^{[20]}$ Maguire et al. ${ }^{[15]}$ found hysterometer placement and uterine depth measurement to be the most painful phase. This inconsistency can be partly explained by differences in participant characteristics and time of IUD insertion between the two studies: while our participants had a history of previous delivery, and IUDs were inserted during the menstruation period, Maguire et al. ${ }^{[15]}$ recruited nulliparous women, and inserted IUDs on non-menstrual days (a dilator was even used to open the cervical os in some cases). Since the internal os is believed to be softest and most open during menstrual days, inserting the hysterometer would be easier and less painful during this period.

In the present research, spraying the cervix with $10 \%$ lidocaine 3 minutes before IUD insertion was found to decrease pain in all phases of the procedure. There were, in fact, significant differences in pain intensity during tenaculum placement, hysterometer insertion and IUD insertion between the intervention and control groups. The difference remained significant at 15 minutes after IUD insertion. The mean (SD) pain intensity during the most painful stage (tenaculum insertion) was 2.20 (1.96) in the lidocaine spray group and 4.25 (1.92) in the control group. Aksoy et al.$^{[18]}$ reported a mean (SD) pain intensity of 1.01 (1.20) in the lidocaine spray group and 3.23 (1.60) in the placebo group. The difference between their study and ours can be attributed to the fact that all their subjects had a history of vaginal delivery, in contrast with the present study. Furthermore, they measured pain at only one stage, immediately after inserting the IUD. By contrast, half of our subjects had no history of vaginal delivery but only of caesarean section, and we assessed the intensity of pain in several stages, including immediately after inserting the IUD. Despite these differences, our finding of a significant decrease in pain intensity during IUD insertion using lidocaine $10 \%$ spray concurs with that of Aksoy et al. ${ }^{[18]}$

Although only one previous study confirmed the efficacy of lidocaine spray in decreasing IUD insertion pain, $10 \%$ lidocaine spray has been shown to be effective in reducing hysterosalpingography pain, ${ }^{[21]}$ hysteroscopy pain ${ }^{[22]}$ and firsttrimester abortion pain. ${ }^{[23]}$

Vanichtantikul and Charoenkwan ${ }^{[24]}$ recommend lidocaine spraying as a practical and effective method to decrease pain during the loop electrosurgical excision procedure. In a clinical trial, Olad-SahebMadarek et al. ${ }^{[25]}$ concluded that the use of lidocaine solution in the uterus was effective in reducing endometrial biopsy pain; however, combining this method with $10 \%$ lidocaine spray to the cervix did not have any additional effects on pain reduction. It seems that different neural pain transmission pathways might be responsible in the cervix v. the uterus: while nerve impulses in the uterine body are transmitted through the 11th and 12th thoracic nerves, the pudendal nerve (derived from anterior roots of the 2nd to 4 th sacral nerves) carries sensations from the cervix. ${ }^{[26,27]}$

Various methods to decrease IUD insertion pain have been tested. Some methods, such as NSAIDs and cervix-softener drugs, including misoprostol, have not been effective in decreasing IUD insertion pain. ${ }^{[9,12,28]}$ Local anaesthesia has also been administered, for example by Maguire et al. ${ }^{[15]}$ Mohammad-Alizadeh-Charandabi et al. ${ }^{[29]}$ and McNicholas et al. ${ }^{[16]}$ applied lidocaine gel to the cervix before IUD insertion and found the method to be ineffective in decreasing IUD insertion pain. Mody et al. ${ }^{[31]}$ used paracervical anaesthesia to decrease IUD insertion pain, but although this invasive method did decrease the insertion pain, it was found to be painful itself. In addition, with this method the injectable substance may be absorbed into the circulation, and in lower doses may be accompanied by side-effects such as flushing, tachycardia and dysphoria. ${ }^{[30]}$

The strength of the present study, in comparison with the similar study by Aksoy et al.. ${ }^{[18]}$ was in the measurement of pain and the

Table 2. Patient pain perception at IUD insertion ( 0 - 10 point scale)

\begin{tabular}{llll}
\hline Variable & Control group, mean (SD) & Intervention group, mean (SD) & $p$-value \\
\hline Background pain (dysmenorrhoea) & $0.47(0.98)$ & $0.55(0.81)$ & $0.38^{*}$ \\
Anticipated pain & $4.35(1.91)$ & $5.02(1.82)$ & $0.06^{*}$ \\
Tenaculum placement & $4.25(1.92)$ & $2.20(1.34)$ & $<0.001^{*}$ \\
Hysterometer placement (uterine sounding) & $3.45(1.95)$ & $1.92(1.40)$ & $<0.001^{*}$ \\
Intrauterine device insertion & $2.95(2.10)$ & $1.30(1.50)$ & $<0.001^{*}$ \\
Pain after 15 minutes & $1.70(1.74)$ & $0.87(1.04)$ & $0.03^{*}$ \\
ANOVA measures (repeated) & - & - & $<0.001$ \\
${ }^{*}$ Mann-Whitney U test. & & & \\
ANOVA = analysis of variance. & & &
\end{tabular}


assessment of the effect of lidocaine spray at several stages of the procedure. However, a limitation was the allocation of participants to intervention (receiving lidocaine spray) and control (routine IUD insertion) groups; perhaps it would have been more reliable to have a third group receiving a placebo instead of lidocaine spray, which should be considered in future studies.

Using lidocaine spray for anaesthesia is non-invasive, cheap, easy and accessible for all medical centres. Despite the benefits observed in the current study, further surveys in different centres and populations should be performed before recommending the application of this method as a part of routine care before IUD insertion in all centres.

Acknowledgments. The present study was extracted from a research project approved by the Islamic Azad University. We hereby thank Urmia Islamic Azad University for supporting this project. We also thank the Research Deputy of the university.

Author contributions. Equal contributions.

Funding. None.

Conflicts of interest. None.

1. Peipert JF, Zhao Q, Allsworth JE, et al. Continuation and satisfaction of reversible contraception Obstetr Gynecol 2011;117(5):1105-1113. https://doi.org/10.1097/AOG.0b013e31821188ad

2. United Nations Department of Economic and Social Affairs, Population Division. World Contraceptive Use. New York: UN, 2012; 2014.

3. Asker C, Stokes-Lampard H, Beavan J, Wilson S. What is it about intrauterine devices that women find unacceptable? Factors that make women non-users: A qualitative study. J Fam Plann Reprod Health Care 2006;32(2):89-94. https://doi.org/10.1783/147118906776276170

4. Weston MR, Martins SL, Neustadt AB, Gilliam ML. Factors influencing uptake of intrauterine devices among postpartum adolescents: A qualitative study. Am J Obstet Gynecol 2012;206(1):el7. https://doi.org/10.1016/j.ajog.2011.06.094

5. Chi IC, Galich LF, Tauber PF, et al. Severe pain at interval IUD insertion: A case-control analysis of patient risk factors. Contraception 1986;34(5):483-495.

6. Dijkhuizen K, Dekkers OM, Holleboom CA, et al. Vaginal misoprostol prior to insertion of an intrauterine device: An RCT. Human Reprod 2011;26(2):323-329. https://doi.org/10.1093/humrep/deq348

7. Ward K, Jacobson JC, Turok DK, Murphy PA. A survey of provider experience with misoprostol to facilitate intrauterine device insertion in nulliparous women. Contraception 2011;84(6):594-599. https://doi.org/10.1016/j.contraception.2011.03.011

8. Allen RH, Bartz D, Grimes DA, Hubacher D, O'Brien P. Interventions for pain with intrauterine device insertion. Cochrane Database Syst Rev 2009;8(3):CD007373. https://doi. org/10.1002/14651858

9. Hubacher D, Reyes V, Lillo S, Zepeda A, Chen PL, Croxatto H. Pain from copper intrauterine device insertion: Randomised trial of prophylactic ibuprofen. Am J Obstet Gynecol 2006;195(5):1272-1277. https://doi.org/j.ajog.2006.08.022

10. Buttram V, Izu A, Henzl MR. Naproxen sodium in uterine pain following intrauterine contraceptive device insertion. Am J Obstet Gynecol 1979;134(5):575-578. https://doi. org/10.1016/0002-9378(79)90844-5

11. Massey SE, Varady JC, Henzl MR. Pain relief with naproxen following insertion of an intrauterine device. J Reprod Med 1974;13:226-31
12. Saav I, Aronsson A, Marions L, Stephansson O, Gemzell-Danielsson K. Cervical priming with sublingual misoprostol prior to insertion of an intrauterine device in nulliparous women: A randomised controlled trial. Human Reprod 2007;22(10):2647-2652. https://doi.org/10.1093/ humrep/dem 244

13. Tolcher R. Intrauterine techniques: Contentious or consensus opinion? J Fam Plan Reprod Health Care 2003;29(1):21-24

14. Oloto EJ, Boromhan DR, Murty JA. Pain and discomfort perception at IUD insertion - effect of short duration, low volume, intracervical application of two percent lignocaine gel (Instillagel). Br J Fam Plan 1996:22:177-80

15. Maguire K, Davis A, Rosario Tejeda L, Westhoff C. Intracervical lidocaine gel for intrauterine device insertion: a randomised controlled trial. Contraception 2012;86(3):214-219. https://doi. org/10.1016/j.contraception.2012.01.005

16. McNicholas CP, Madden T, Zhao Q, Secura G, Allsworth JE, Peipert JF. Cervical lidocaine for IUD insertional pain: A randomised controlled trial. Am J Obstet Gynecol 2012;207:e1-6. https://doi. org/10.1016/.j.ajog.2012.09.018

17. Mody SK, Kiley J, Rademaker A, Gawron L, Stika C, Hammond, C. Pain control for intrauterine device insertion: A randomised trial of $1 \%$ lidocaine paracervical block. Contraception 2012;86(6):704-709. https://doi.org/10.1016/j.contraception.2012.06.004

18. Aksoy H, Aksoy U, Ozyurt S, Açmaz G, Babayigit M. Lidocaine spray 10\% to the cervix reduces pain during intrauterine device insertion: A double blind-randomised controlled trial. J Fam Plan Reprod Health Care 2016;42(2):83-87. https://doi.org/10.1136/jfprhc-2014-100917

19. Gemzell-Danielsson K, Mansour D, Fiala C, Kaunitz AM, Bahamondes L. Management of pain associated with the insertion of intrauterine contraceptives. Human Reprod 2013;19(4):419-427. https://doi.org/10.1093/humupd/dmt022

20. Seamark C. Is the fitting of an intrauterine contraceptive device a painful experience? Br J Fam Plan 1993;19:229-231.

21. Karasahin E, Alanbay I, Keskin U, Gezginc K, Baser I. Lidocaine 10\% spray reduces pain during hysterosalpingography: A randomised controlled trial. J Obstet Gynaecol Res 2009;35(2):354-358. https://doi.org/10.1111/j.1447-0756.2008.00938.x

22. Soriano D, Ajaj S, Chuong T, Deval B, Fauconnier A, Darai E. Lidocaine spray and outpatient hysteroscopy: Randomised placebo-controlled trial. Obstet Gynecol 2000;96(5 pt 1):661-664.

23. Karasahin KE, Alanbay I, Ercan CM, Mesten Z, Simsek C, Baser I. Lidocaine spray in addition to paracervical block reduces pain during first-trimester surgical abortion: A placebo-controlled clinical trial. Contraception 2011;83(4):362-366. https://doi.org/10.1016/j.contraception.2010.07.025

24. Vanichtantikul A, Charoenkwan K. Lidocaine spray compared with submucosal injection for reducing pain during loop electrosurgical excision procedure: A randomised controlled trial. Obstet Gynecol 2013;122(3):553-557. https://doi.org/10.1097/AOG.0b013e31829d888e

25. Olad-Saheb-Madarek E, Garabaghi P, Behjati F, Azadi A, Seidhejazie M. Role of local anaesthetics on pain relief in endometrial biopsy: Randomised clinical trial. Int J Women's Health Reprod Sc 2014;2(3):58-66.

26. Cunningham F, Leveno K, Bloom S, Hauth J. Williams Obstetrics. New York: McGraw-Hill, 2014.

27. Liberty G, Gal M, Halevey-Shalem T, Michaelson-Cohen R, Galoyan N, Hyman J. Lidocaineprilocaine (EMLA) cream as analgesia for hysterosalpingography: A prospective, randomised, controlled, double blinded study. Human Reproduction 2007;22(5):1335-1339. https://doi. org/10.1093/humrep/del517

28. Chor J, Bregand-White J, Golobof A, Harwood B, Cowett A. Ibuprofen prophylaxis for levonorgestrel-releasing intrauterine system insertion: A randomised controlled trial. Contraception 2012;85(6):558-562. https://doi.org/10.1016/j.contraception.2011.10.015

29. Mohammad-Alizadeh-Charandabi S, Seidi S, Kazemi F. Effect of lidocaine gel on pain from copper IUD insertion: a randomised double-blind controlled trial. Indian J Med Sci 2010;64(8):349-355. https://doi.org/10.4103/0019-5359.100337

30. Nadelberg R. Paracervical Block. Boston: Boston Scientific Corporation, 2007.

31. Mody SK, Farala JP, Jimenez B, Nishikawa M, Ngo LL. Paracervical block for intrauterine device placement among nulliparous women: A randomised controlled trial. Obstet Gynecol 2018;132(3):575-582. https://doi.org/10.1097/AOG.0000000000002790 